\title{
SOME SUFFICIENT CONDITIONS FOR HEEGAARD GENERA TO BE ADDITIVE UNDER ANNULUS SUM
}

\author{
FENGLING LI* ${ }^{*}$ FENGCHUN LEI ${ }^{\dagger}$ and GUOQIU YANG ${ }^{\ddagger}$
}

\begin{abstract}
Let $M_{i}$ be a compact orientable 3-manifold, and $A_{i}$ an incompressible annulus on a component $F_{i}$ of $\partial M_{i}, i=1,2$. Suppose $A_{1}$ is separating on $F_{1}$ and $A_{2}$ is non-separating on $F_{2}$. Let $M$ be the annulus sum of $M_{1}$ and $M_{2}$ along $A_{1}$ and $A_{2}$. In the present paper we show that if $M_{i}$ has a Heegaard splitting $V_{i} \cup_{S_{i}} W_{i}$ with Heegaard distance $d\left(S_{i}\right) \geq 2 g\left(M_{i}\right)+5$ for $i=1,2$, then $g(M)=g\left(M_{1}\right)+g\left(M_{2}\right)$. Moreover, when $g\left(F_{2}\right) \geq 2$, the minimal Heegaard splitting of $M$ is unique up to isotopy.
\end{abstract}

\section{Introduction}

Let $M_{i}$ be a compact connected orientable bordered 3-manifold, and $A_{i}$ an incompressible annulus on $\partial M_{i}, i=1,2$. Let $h: A_{1} \rightarrow A_{2}$ be a homeomorphism. The manifold $M$ obtained by gluing $M_{1}$ and $M_{2}$ along $A_{1}$ and $A_{2}$ via $h$ is called an annulus sum of $M_{1}$ and $M_{2}$ along $A_{1}$ and $A_{2}$, and is denoted by $M_{1} \cup_{h} M_{2}$ or $M_{1} \cup_{A_{1}=A_{2}} M_{2}$.

Let $V_{i} \cup_{S_{i}} W_{i}$ be a Heegaard splitting of $M_{i}, i=1,2$, and $M=M_{1} \cup_{A_{1}=A_{2}}$ $M_{2} . M$ has a natural Heegaard splitting $V \cup_{S} W$ induced from $V_{1} \cup_{S_{1}} W_{1}$ and $V_{2} \cup_{S_{2}} W_{2}$ with genus $g(S)=g\left(S_{1}\right)+g\left(S_{2}\right)$ (refer to Schultens [15]). Let $g(M)$ be the minimal genus among all Heegaard surfaces of $M$. Then we always have $g(M) \leq g\left(M_{1}\right)+g\left(M_{2}\right)$.

Some sufficient conditions for tunnel number of knots not to go down under connected sum are given in [17]. When both $A_{1}$ and $A_{2}$ are non-separating on the corresponding boundary component, there are some sufficient conditions for the equality $g(M)=g\left(M_{1}\right)+g\left(M_{2}\right)$ to hold, see [2], [8].

In the present paper, we consider the case that $A_{1}$ is separating on $F_{1}$ (so necessarily $g\left(F_{1}\right)>1$ ) and $A_{2}$ is non-separating on $F_{2}$. The following are the main results of the paper.

\footnotetext{
* Supported in part by two grants of NSFC (No. 11101058 and No. 11329101).

$\dagger$ Supported in part by a key grant of NSFC (No. 10931005) and a grant of NSFC (No. 11329101).

\$ Supported in part by two grants of NSFC (No. 11001065 and No. 11071106).

Received 2 July 2012, in final form 24 June 2013.
} 
TheORem 1.1. Let $M$ be an irreducible 3-manifold, and A a properly embedded essential annulus separating $M$ into $M_{1}$ and $M_{2}$. Suppose $A_{i}=A$ lies in an incompressible boundary component $F_{i}$ of $M_{i}$ for $i=1,2$, and $A_{1}$ is separating on $F_{1}$ and $A_{2}$ is non-separating on $F_{2}$. If $M_{i}$ has a Heegaard splitting $V_{i} \cup_{S_{i}} W_{i}$ withd $\left(S_{i}\right) \geq 2 g\left(M_{i}\right)+5$ for $i=1,2$. Then $g(M)=g\left(M_{1}\right)+g\left(M_{2}\right)$.

TheORem 1.2. Let $M$ be an irreducible 3-manifold, and A a properly embedded essential annulus separating $M$ into $M_{1}$ and $M_{2}$. Suppose $A_{i}=A$ lies in an incompressible boundary component $F_{i}$ of $M_{i}$ for $i=1,2$, and $A_{1}$ is separating on $F_{1}$ and $A_{2}$ is non-separating on $F_{2}$. Suppose $M_{i}$ has a Heegaard splitting $V_{i} \cup_{S_{i}} W_{i}$ with $d\left(S_{i}\right) \geq 2 g\left(M_{i}\right)+5$ for $i=1,2$, then

(1) If $g\left(F_{2}\right) \geq 2$, then the minimal Heegaard splitting of $M$ is unique up to isotopy;

(2) If $g\left(F_{2}\right)=1$, then there are at most two minimal Heegaard splittings of M up to isotopy.

Note that papers [8] and [17] only consider the case that both $A_{1}$ and $A_{2}$ are non-separating. In [2], the bound of the Heegaard distance for the additivity to hold is " $d\left(S_{i}\right) \geq 2\left(g\left(M_{1}\right)+g\left(M_{2}\right)\right), i=1,2$ ", in other words, the bound of $d\left(S_{1}\right)\left(d\left(S_{2}\right)\right.$, resp.) of $M_{1}\left(M_{2}\right.$, resp. ) not only relates to the genus of $M_{1}\left(M_{2}\right.$, resp.), but also relates to the genus of the other manifold $M_{2}\left(M_{1}\right.$, resp.). While in our results, the bound is " $d\left(S_{i}\right) \geq 2 g\left(M_{i}\right)+5, i=1,2$ ", that is, $d\left(S_{1}\right)$ only relates to $g\left(M_{1}\right)$ and $d\left(S_{2}\right)$ only relates to $g\left(M_{2}\right)$. And with the weaker assumptions, we obtain the stronger conclusion that the minimal Heegaard splitting of $M$ is in some sense unique. Hence we remark that the situations here are quite different from those in [2], [8] and [17], and the arguments there are not applicable to the main cases here.

The paper is organized as follows. In Section 2, we review some preliminaries which will be used later. In Section 3, we give the proof of the main results.

\section{Preliminaries}

In this section, we will review some fundamental facts on surfaces in 3manifolds. Definitions and terms which have not been defined are all standard, refer to, for example, [5].

A Heegaard splitting of a 3-manifold $M$ is a decomposition $M=V \cup_{S} W$ in which $V$ and $W$ are compression bodies and $S=V \cap W=\partial_{+} V=\partial_{+} W$ is the Heegaard surface. $V \cup_{S} W$ is said to be weakly reducible if there are essential disks $D_{1} \subset V$ and $D_{2} \subset W$ with $\partial D_{1} \cap \partial D_{2}=\emptyset$. Otherwise, $V \cup_{S} W$ is strongly irreducible (see [1]). 
Suppose $M=V \cup_{S} W$ is a Heegaard splitting. Let $\mathscr{C}_{V}(S)\left(\mathscr{C}_{W}(S)\right.$, resp. $)$, denote the set of all simple closed curves on $S$ which bound essential disks in $V\left(W\right.$, resp.). Define $d(S)=\min \left\{d(\alpha, \beta) \mid \alpha \in \mathscr{C}_{V}(S), \beta \in \mathscr{C}_{W}(S)\right\}$, where $d(\alpha, \beta)$ is measured in the curve complex $\mathscr{C}(S)$ of $S$ (see [4]).

Let $F$ be a surface properly embedded in $M$. We say that $F$ is $\partial$-parallel if $F$ cuts off a 3-manifold homeomorphic to $F \times[0,1]$ from $M$. A properly embedded surface is essential if it is incompressible and not $\partial$-parallel.

Let $P$ be a properly embedded separating surface in a 3-manifold $M$ which cuts $M$ into two 3-manifolds $M_{1}$ and $M_{2}$. Then $P$ is bicompressible if $P$ has compressing disks in both $M_{1}$ and $M_{2} . P$ is strongly irreducible if it is bicompressible and each compressing disk in $M_{1}$ meets each compressing disk in $M_{2}$.

Now let $P$ be a closed bicompressible surface in an irreducible 3-manifold $M$. By maximally compressing $P$ in both sides of $P$ and removing any resulting 2-sphere components, we obtain two surfaces that we denote by $P_{+}$and $P_{-}$. Let $H_{1}^{P}$ denote the closure of the region that lies between $P$ and $P_{+}$and similarly define $H_{2}^{P}$ to denote the closure of the region that lies between $P$ and $P_{-}$. Then $H_{1}^{P}$ and $H_{2}^{P}$ are compression bodies. If $P$ is strongly irreducible in $M$, then the Heegaard splitting $H_{1}^{P} \cup_{P} H_{2}^{P}$ is strongly irreducible. Two strongly irreducible surfaces $P$ and $Q$ are said to be well-separated in $M$ if $H_{1}^{P} \cup_{P} H_{2}^{P}$ may be isotoped to be disjoint from $H_{1}^{Q} \cup_{Q} H_{2}^{Q}$.

Scharlemann and Thompson ([13]) showed that any irreducible and $\partial$ irreducible Heegaard splitting $M=V \cup_{S} W$ has an untelescoping as

$$
V \cup_{S} W=\left(V_{1} \cup_{S_{1}} W_{1}\right) \cup_{F_{1}}\left(V_{2} \cup_{S_{2}} W_{2}\right) \cup_{F_{2}} \ldots \cup_{F_{m-1}}\left(V_{m} \cup_{S_{m}} W_{m}\right),
$$

such that each $V_{i} \cup_{S_{i}} W_{i}$ is a strongly irreducible Heegaard splitting with $F_{i}=\partial_{-} W_{i} \cap \partial_{-} V_{i+1}, 1 \leq i \leq m-1, \partial_{-} V_{1}=\partial_{-} V, \partial_{-} W_{m}=\partial_{-} W$, and for each $i$, each component of $F_{i}$ is a closed incompressible surface of positive genus, and only one component of $M_{i}=V_{i} \cup_{S_{i}} W_{i}$ is not a product. It is easy to see that when $m \geq 2, g(S) \geq g\left(S_{i}\right)+1 \geq g\left(F_{i}\right)+2$ for each $i$. From $V_{1} \cup_{S_{1}} W_{1}, \ldots, V_{m} \cup_{S_{m}} W_{m}$, we can get a Heegaard splitting of $M$ by a process called amalgamation (see [16]).

The following are some basic facts and results on Heegaard splittings.

Lemma 2.1 ([11]). Suppose $(Q, \partial Q) \subset(M, \partial M)$ is an essential surface and $Q^{\prime}$ is the result of $\partial$-compressing $Q$ in $M$. Then $Q^{\prime}$ is essential.

LEMMA 2.2 ([15], [9]). Let $F$ be an incompressible surface (not a 2-sphere, a 2-disk or a projective plane) properly embedded in $M=V \cup_{S} W$. If $V \cup_{S} W$ is strongly irreducible, then $F$ can be isotoped so that $S \cap F$ consists of loops that are essential in both $F$ and $S$. 
The following Lemma is a well known fact (see [15]).

Lemma 2.3. An incompressible surface $F$ in a compression body $V$ with $\partial F \subset \partial_{+} V$ cuts $V$ into compression bodies.

Lemma 2.4 ([9]). Let $V$ be a non-trivial compression body and $\mathscr{A}$ be a disjoint union of essential annuli properly embedded in $V$. Then there is an essential disk $D$ in $V$ with $D \cap \mathscr{A}=\emptyset$.

Lemma 2.5 ([3], [11]). Let $V \cup_{S} W$ be a Heegaard splitting of $M$ and $F$ be a properly embedded incompressible surface (maybe not connected) in $M$. Then either any component of $F$ is $\partial$-parallel in $M$ or $d(S) \leq 2-\chi(F)$.

Lemma 2.6 ([14]). Let $P$ and $Q$ be strongly irreducible connected closed separating surfaces in a 3-manifold $M$. Then either

(1) $P$ and $Q$ are well-separated,

(2) $P$ and $Q$ are isotopic, or

(3) $d(P) \leq 2 g(Q)$.

Lemma 2.7 ([6]). Let $M=V \cup_{S} W$ be a Heegaard splitting with $d(S)>$ $2 g(M)$. Then $V \cup_{S} W$ is the unique minimal Heegaard splitting of $M$ up to isotopy.

Lemma 2.8 ([12]). Let $V$ be a non-trivial compression body and $\mathscr{A}$ be a disjoint union of incompressible annuli properly embedded in $V$. If $U$ is a component of $V \backslash \mathscr{A}$ with $U \cap \partial_{-} V \neq \emptyset$, then $\chi\left(U \cap \partial_{-} V\right) \geq \chi\left(U \cap \partial_{+} V\right)$.

LEMMA 2.9 ([8], [17]). Let $N$ be a compact orientable 3-manifold which is not a compression body, and $F=\partial N$. Suppose $Q$ is a properly embedded connected separating surface in $N$ with $\partial Q$ essential in $F$, and $Q$ cuts $N$ into two compression bodies $N_{1}$ and $N_{2}$ with $Q=\partial_{+} N_{1} \cap \partial_{+} N_{2}$ and $F \cap N_{2}$ a collection of annuli. If $Q$ is compressible in both $N_{1}$ and $N_{2}$, and $Q$ can be compressed to $Q^{*}$ in $N_{1}$ such that any component of $Q^{*}$ is $\partial$-parallel in $N$, then $N$ has a Heegaard splitting $V \cup_{S} W$ with $g(S)=1-\frac{1}{2} \chi(Q)$ and $d(S) \leq 2$.

\section{The proof of the main results}

In $M=M_{1} \cup_{h} M_{2}$, let $A=A_{2}=h\left(A_{1}\right)$ and $F_{i}$ be the incompressible boundary component of $M_{i}$ in which $A_{i}$ lies, $i=1,2$. We denote the two components of $F_{1}-$ int $A$ by $F_{1}^{1}$ and $F_{1}^{2}$, and let $F_{3}=F_{1}^{1} \cup\left(F_{2}-\right.$ int $\left.A\right) \cup F_{1}^{2}$, then $F_{3}$ is a boundary component of $M$. Let $I=[0,1]$ and $F_{i} \times I$ be a regular neighborhood of $F_{i}$ in $M_{i}$ with $F_{i}=F_{i} \times\{0\}$. We denote the surface $F_{i} \times\{1\}$ by $F^{i}$, it's clear that $F^{i}$ is incompressible in $M_{i}$. Let $M^{i}=M_{i}-F_{i} \times[0,1)$ for $i=1,2$, and $M^{0}=F_{1} \times I \cup_{A} F_{2} \times I$. Then $M=M^{1} \cup_{F^{1}} M^{0} \cup_{F^{2}} M^{2}$. Note 
that $M^{0}$ contains three boundary components $F^{1}, F^{2}$ and $F_{3}$. By [2] Lemma 2.3, $M^{0}$ contains only two essential surfaces up to isotopy, $F_{1}^{*}$ and $F_{2}^{*}$, say, where $F_{1}^{*}=X_{1} \cup X_{2} \cup X_{3}$ such that $X_{1}$ and $X_{3}$ are isotopic to $F_{1}^{1}$, and $X_{2}$ is a copy of $F_{2}-i n t A$. And $F_{2}^{*}=Y_{1} \cup Y_{2} \cup Y_{3}$ such that $Y_{1}$ and $Y_{3}$ are isotopic to $F_{1}^{2}$, and $Y_{2}$ is a copy of $F_{2}-i n t A$.

Lemma 3.1. Let $M^{1}, M^{0}, F^{1}$ and $F^{2}$ be as above. If $M_{1}$ has a Heegaard splitting $V_{1} \cup_{S_{1}} W_{1}$ with $d\left(S_{1}\right) \geq 2 g\left(M_{1}\right)+5$, then any minimal Heegaard splitting of $M^{1} \cup_{F^{1}} M^{0}$ is the amalgamation of minimal Heegaard splittings of $M^{1}$ and $M^{0}$, and $g\left(M^{1} \cup_{F^{1}} M^{0}\right)=g\left(M_{1}\right)+g\left(F_{2}\right)$.

Proof. Now suppose that $V \cup_{S} W$ is a minimal Heegaard splitting of $M^{1} \cup_{F^{1}}$ $M^{0}$ with $F_{3} \subset \partial_{-} V$. Since $M^{1} \cup_{F^{1}} M^{0}=M_{1} \cup_{A}\left(F_{2} \times I\right)$, by a result of Schultens [15], $g(S) \leq g\left(M_{1}\right)+g\left(F_{2}\right)$.

First we show that $M_{1}$ is not a compression body. Otherwise, $M_{1}$ is either a (closed surface) $\times I$ or $\partial$-reducible. In each case, $M_{1}$ cannot have a Heegaard splitting $V_{1} \cup_{S_{1}} W_{1}$ with distance $d\left(S_{1}\right) \geq 2 g\left(M_{1}\right)+5$.

We will show that $V \cup_{S} W$ is weakly reducible. So suppose for contradiction that $V \cup_{S} W$ is strongly irreducible. First note that $S \cap A \neq \emptyset$ since $F^{1}$ is essential in $M$ and there is no closed essential surface in a compression body. By Lemma 2.2, we may assume that each component of $S \cap A$ is essential in both $S$ and $A$, and $|S \cap A|$ is minimal. Since $A$ is an essential annulus in $M$, by Lemma 2.4, $S \backslash A$ has compressing disks in both $V$ and $W$. If there are distinct components of $S \backslash A$, say $P_{1}$ and $P_{2}$, such that $P_{1}$ has a compressing disk $D_{V}$ in $V$ and $P_{2}$ has a compressing disk $D_{W}$ in $W$, but $P_{1} \neq P_{2}$, then we have $\partial D_{V} \cap \partial D_{W}=\emptyset$, contradicting the strong irreducibility of $V \cup_{S} W$. Hence we assume that all components of $S \backslash A$ are incompressible in $M \backslash A$ except exactly one bicompressible component.

Claim 1. $\chi\left(S \cap M_{1}\right) \leq-2 g\left(M_{1}\right)$.

PRoof. Now if $S \cap M_{1}$ is incompressible, then it is essential in $M_{1}$. Otherwise, any component of $S \cap M_{1}$ is $\partial$-parallel in $M_{1}$, which means that $M_{1}$ is a compression body, a contradiction. By Lemma $2.5,2-\chi\left(S \cap M_{1}\right) \geq d\left(S_{1}\right) \geq$ $2 g\left(M_{1}\right)+5$, thus $\chi\left(S \cap M_{1}\right) \leq-3-2 g\left(M_{1}\right)$.

Now suppose $S \cap M_{1}$ is bicompressible. We denote the bicompressible component of $S \cap M_{1}$ by $P$. In fact, $P$ is strongly irreducible in $M_{1}$. We claim that $\chi(P) \leq-2$. If not, $P$ is either a disk, an annulus, a pair of pants, or a once punctured torus. In each case we conclude that a component of $\partial P$ bounds a disk in $M_{1}$, therefore $A$ is compressible in $M_{1}$, a contradiction. If there exists an incompressible component $Q$ of $S \cap M_{1}$ which is essential in $M_{1}$, by Lemma $2.5,2-\chi(Q) \geq d\left(S_{1}\right) \geq 2 g\left(M_{1}\right)+5$, and then $\chi\left(S \cap M_{1}\right) \leq$ $\chi(Q)+\chi(P) \leq-5-2 g\left(M_{1}\right)$. Hence in the following we may assume that 
the incompressible components of $S \cap M_{1}$ are all $\partial$-parallel in $M_{1}$. Let $P^{V}$ be the surface obtained by maximally compressing $P$ in $V$ and removing all possible 2 -sphere components. Since $P$ is strongly irreducible, we can see that, by the No Nesting Lemma ([10]) and [10, Lemma 5.5], $P^{V}$ is incompressible in $M_{1}$. Now if $P^{V}$ is essential in $M_{1}$, by Lemma $2.5,2-\chi\left(P^{V}\right) \geq d\left(S_{1}\right) \geq$ $2 g\left(M_{1}\right)+5$, and then $\chi\left(S \cap M_{1}\right) \leq \chi(P) \leq \chi\left(P^{V}\right)-2 \leq-5-2 g\left(M_{1}\right)$. Therefore we may assume that each component of $P^{V}$ is $\partial$-parallel in $M_{1}$.

Since $A$ is an essential annulus in $M$ and by Lemma 2.3, each component of $V \cap M_{1}$ and $W \cap M_{1}$ is a compression body. Let $U_{1}$ be the component of $V \cap M_{1}$ containing $P$ and $U_{2}$ be the component of $W \cap M_{1}$ containing $P$. Since any component of $S \cap M_{1}$ other than $P$ is $\partial$-parallel, $U_{1} \cup_{P} U_{2} \cong M_{1}$ and $\partial_{+} U_{1} \cap \partial_{+} U_{2}=P$. Since $M_{1}$ is not a compression body and $A$ is an annulus, by Lemma 2.9, there exists a Heegaard surface $S^{1}$ of $M_{1}$ with $d\left(S^{1}\right) \leq 2$ and $g\left(S^{1}\right) \leq 1-\frac{1}{2} \chi(P)$. Since $d\left(S^{1}\right) \leq 2$, by Lemma $2.7, S^{1}$ is not isotopic to the unique minimal Heegaard surface $S_{1}$ of $M_{1}$, we have $g\left(S^{1}\right) \geq g\left(M_{1}\right)+1$. Hence $\chi\left(S \cap M_{1}\right) \leq \chi(P) \leq 2-2 g\left(S^{1}\right) \leq-2 g\left(M_{1}\right)$.

This completes the proof of Claim 1.

Claim 2. $\chi\left(S \cap\left(F_{2} \times I\right)\right) \leq \chi\left(F_{2}\right)$.

PROOF. Since $A$ is an essential annulus in $M$, if we denote the component of $V \cap\left(F_{2} \times I\right)$ or $W \cap\left(F_{2} \times I\right)$ which contains $F^{2}$ by $U$, by Lemma 2.8, we have $\chi\left(S \cap\left(F_{2} \times I\right)\right) \leq \chi\left(U \cap\left(S \cap\left(F_{2} \times I\right)\right)\right) \leq \chi\left(U \cap F^{2}\right)=\chi\left(F_{2}\right)$.

This completes the proof of Claim 2.

Now by the proof of Claim 1, we know that if $S \cap M_{1}$ is incompressible in $M_{1}$ then it is essential in $M_{1}$, and therefore by Lemma $2.5, \chi\left(S \cap M_{1}\right) \leq$ $-3-2 g\left(M_{1}\right)$. Then $2 g(S)=2-\chi\left(S \cap M_{1}\right)-\chi\left(S \cap\left(F_{2} \times I\right)\right) \geq 2 g\left(M_{1}\right)+$ $2 g\left(F_{2}\right)+3$, a contradiction. Hence $S \cap M_{1}$ is bicompressible in $M_{1}$ and $\chi(S \cap$ $\left.M_{1}\right) \leq-2 g\left(M_{1}\right)$. Note that $S$ is a strongly irreducible Heegaard surface of $M$, so $S \cap\left(F_{2} \times I\right)$ is incompressible in $F_{2} \times I$. By Lemma 2.1, $S \cap\left(F_{2} \times I\right)$ is $\partial$-parallel in $F_{2} \times I$ since any incompressible and $\partial$-incompressible surface in a trivial compression body is just a spanning annulus.

If $\chi\left(S \cap\left(F_{2} \times I\right)\right)<\chi\left(F_{2}\right)$, then $2 g(S)=2-\chi\left(S \cap M_{1}\right)-\chi\left(S \cap\left(F_{2} \times I\right)\right)>$ $2 g\left(M_{1}\right)+2 g\left(F_{2}\right)$, a contradiction. Thus $\chi\left(S \cap\left(F_{2} \times I\right)\right)=\chi\left(F_{2}\right)$. Now if $\chi\left(F_{2}\right)=0$, i.e., $g\left(F_{2}\right)=1$, then $g(S) \leq g\left(M_{1}\right)+1$. Since $S$ is a Heegaard surface of $M_{1} \cup_{A} F_{2} \times I$ while $S_{1}$ is a Heegaard surface of $M_{1}, S$ and $S_{1}$ are not well-separated, furthermore, $S$ is not isotopic to $S_{1}$. Then by Lemma 2.6, $d\left(S_{1}\right) \leq 2 g(S) \leq 2 g\left(M_{1}\right)+2<2 g\left(M_{1}\right)+5$, a contradiction. So $g\left(F_{2}\right)>1$ and $S \cap\left(F_{2} \times I\right)$ has one and only one component. Since $A_{2}$ is non-separating, we have that $|S \cap A|=2$.

We take an essential arc $\alpha$ in $S \cap\left(F_{2} \times I\right)$ such that $\alpha$ is adjacent to the two components of $S \cap A$. Let $N^{*}=N\left((S \cap A) \cup \alpha, S \cap\left(F_{2} \times I\right)\right)$ be the regular 
neighborhood of $(S \cap A) \cup \alpha$ in $\left.S \cap\left(F_{2} \times I\right)\right)$, and denote the component of $\partial N^{*}$ which is not a component of $S \cap A$ by $\beta^{*}$, from the construction of regular neighborhood we may assume that $\beta^{*} \subset F_{2} \times\left\{\frac{1}{2}\right\}$ in $F_{2} \times I$. Set $A^{*}=\beta \times I$ with $\beta \subset F_{2} \backslash A_{2}$ and $\beta^{*}=\beta \times\left\{\frac{1}{2}\right\}$. Denote the component of $\left(F_{2} \backslash A_{2}\right) \backslash \beta$ which doesn't contain $\partial A_{2}$ by $F^{*}$. By substituting $F^{*} \times I$ with (a disk) $\times I$, we get a 3-manifold $M^{\prime}$ with a Heegaard surface $S^{\prime}$ induced from the Heegaard surface $S$ of $M^{1} \cup_{F^{1}} M^{0}$. Since $S$ is strongly irreducible, $S^{\prime}$ is strongly irreducible with $g\left(S^{\prime}\right) \leq g\left(M_{1}\right)+1$. Then by Lemma 2.6, $d\left(S_{1}\right) \leq 2 g\left(S^{\prime}\right) \leq 2 g\left(M_{1}\right)+2<2 g\left(M_{1}\right)+5$, a contradiction.

Hence $V \cup_{S} W$ is weakly reducible. Then $V \cup_{S} W$ has an untelescoping as $V \cup_{S} W=\left(V_{1}^{\prime} \cup_{S_{1}^{\prime}} W_{1}^{\prime}\right) \cup_{H_{1}} \ldots \cup_{H_{n-1}}\left(V_{n}^{\prime} \cup_{S_{n}^{\prime}} W_{n}^{\prime}\right)$, where $n \geq 2$, and each component of $H_{i}, 1 \leq i \leq n-1$, is a closed essential surface in $M^{1} \cup_{F^{1}} M^{0}$. Let $\mathscr{H}=\bigcup_{i=1}^{n-1} H_{i}$. We may assume that any component of $\mathscr{H} \cap A$ is essential in both $A$ and $\mathscr{H}$, and $|\mathscr{H} \cap A|$ is minimal. If $\mathscr{H} \cap A \neq \emptyset$, let $H$ be a component of $\mathscr{H}$ with $H \cap A \neq \emptyset$. If $H \cap M_{1}$ is essential in $M_{1}$ and $H \cap\left(F_{2} \times I\right)$ is $\partial$-parallel in $F_{2} \times I$, by Lemma 2.5, $2-\chi\left(H \cap M_{1}\right) \geq d\left(S_{1}\right) \geq 2 g\left(M_{1}\right)+5$, $\chi\left(H \cap\left(F_{2} \times I\right)\right) \leq \chi\left(F_{2}\right)$, and then $2 g(S) \geq 2 g(H)+4=6-\chi\left(H \cap M_{1}\right)-$ $\chi\left(H \cap\left(F_{2} \times I\right)\right) \geq 2 g\left(M_{1}\right)+2 g\left(F_{2}\right)+4$, a contradiction.

Hence if $H \cap A \neq \emptyset, H \cap M_{1}$ is $\partial$-parallel in $M_{1}$ and $H \cap\left(F_{2} \times I\right)$ is $\partial$-parallel in $F_{2} \times I$. Then $H$ is an essential closed surface in $M^{0}$, hence $H$ is isotopic to either $F_{1}^{*}$ or $F_{2}^{*}$. We may assume that $H$ is isotopic to $F_{1}^{*}$.

If there is no component of $\mathscr{H}$ in $M_{1}$, we denote the Heegaard splitting in the untelescoping between $F_{1}^{*}$ and $F_{3}$ by $N_{1}=V_{1}^{\prime} \cup_{S_{1}^{\prime}} W_{1}^{\prime}$. Since $A$ is essential in $M$, it is essential in $N_{1}$. Note that $N_{1} \cap M_{1} \cong M_{1}$ and $N_{1} \cap M_{2} \cong F_{2} \times I$. Since $V_{1}^{\prime} \cup_{S_{1}^{\prime}} W_{1}^{\prime}$ is strongly irreducible, by Claim 1 and Claim 2 applied to this splitting we have $\chi\left(S_{1}^{\prime} \cap M_{1}\right) \leq-2 g\left(M_{1}\right), \chi\left(S_{1}^{\prime} \cap\left(F_{2} \times I\right)\right) \leq \chi\left(F_{2}\right)$. Then $2 g(S) \geq 4-\chi\left(S_{1}^{\prime}\right) \geq 2 g\left(M_{1}\right)+2 g\left(F_{2}\right)+2$, a contradiction.

Hence there is some component of $\mathscr{H}$ in $M_{1}$. We denote the outermost one by $F^{*}$, and suppose $F^{*}$ is essential in $M_{1}$. We denote the Heegaard splitting in the untelescoping between $F^{*}$ and $F_{3}$ by $N_{1}=V_{1}^{\prime} \cup_{S_{1}^{\prime}} W_{1}^{\prime}$.

Claim 3. $\chi\left(S_{1}^{\prime} \cap M_{1}\right) \leq-3-2 g\left(M_{1}\right)$.

Proof. Since $F^{*}$ is essential in $M_{1}$, by Lemma $2.52-\chi\left(F^{*}\right) \geq d\left(S_{1}\right) \geq$ $2 g\left(M_{1}\right)+5$. If we denote the component of $V_{1}^{\prime} \cap M_{1}$ or $W_{1}^{\prime} \cap M_{1}$ which contains the essential component $F^{*}$ by $U$, since $A$ is an essential annulus in $M$, by Lemma 2.8, we have $\chi\left(S_{1}^{\prime} \cap M_{1}\right) \leq \chi\left(U \cap\left(S_{1}^{\prime} \cap M_{1}\right)\right) \leq \chi\left(U \cap F^{*}\right) \leq$ $\chi\left(F^{*}\right) \leq-3-2 g\left(M_{1}\right)$.

This completes the proof of Claim 3.

Now by Claim 2, we have $\chi\left(S_{1}^{\prime} \cap\left(F_{2} \times I\right)\right) \leq \chi\left(F_{2}\right)$. Then $2 g(S) \geq$ $4-\chi\left(S_{1}^{\prime}\right) \geq 2 g\left(M_{1}\right)+2 g\left(F_{2}\right)+5$, a contradiction. 
Hence $S_{1}^{\prime} \cap A=\emptyset$, and since $F^{*}$ is the outermost component of $\mathscr{H}$ in $M_{1}$, we know that some component of $\mathscr{H}$ must be parallel to $F^{1}$ in $M_{1}$.

Then we get a generalized Heegaard splitting as: $V \cup_{S} W=\left(V_{1}^{\prime} \cup_{S_{1}^{\prime}} W_{1}^{\prime}\right) \cup_{H_{1}}$ $\left(V_{2}^{\prime} \cup_{S_{2}^{\prime}} W_{2}^{\prime}\right.$ ), and $H_{1}$ is isotopic to $F^{1}$. We may further assume that $V_{1}^{\prime} \cup_{S_{1}^{\prime}} W_{1}^{\prime}$ is a Heegaard splitting of $M^{1}$, and $V_{2}^{\prime} \cup_{S_{2}^{\prime}} W_{2}^{\prime}$ is a Heegaard splitting of $M^{0}$. Since $A$ is separating on $F_{1}$ and non-separating on $F_{2}, M^{0}$ contains only three boundary components $F^{1}, F^{2}$ and $F_{3}$. Note that $g\left(F_{3}\right)=g\left(F_{1}\right)+g\left(F_{2}\right)-1$, hence $g\left(S_{2}^{\prime}\right) \geq g\left(M^{0}\right) \geq g\left(F_{1}\right)+g\left(F_{2}\right)$. Then we have $g(S)=g\left(S_{1}^{\prime}\right)+g\left(S_{2}^{\prime}\right)-$ $g\left(H_{1}\right) \geq g\left(M_{1}\right)+g\left(F_{2}\right)$, and equality holds if and only if $g\left(S_{1}^{\prime}\right)=g\left(M_{1}\right)$, $g\left(S_{2}^{\prime}\right)=g\left(F_{1}\right)+g\left(F_{2}\right)$. Combining this with Schultens' result we see that $g(S)=g\left(M_{1}\right)+g\left(F_{2}\right)$, and therefore the previous inequality is an equality, implying that $g\left(S_{1}^{\prime}\right)=g\left(M_{1}\right)$ and $g\left(S_{2}^{\prime}\right)=g\left(F_{1}\right)+g\left(F_{2}\right)$. Hence $V \cup_{S} W$ is the amalgamation of minimal Heegaard splittings of $M^{1}$ and $M^{0}$.

LEMMA 3.2. Let $M^{2}, M^{0}$ and $F^{2}$ be as above. If $M_{2}$ has a Heegaard splitting $V_{2} \cup_{S_{2}} W_{2}$ with $d\left(S_{2}\right) \geq 2 g\left(M_{2}\right)+5$, then any minimal Heegaard splitting of $M^{2} \cup_{F^{2}} M^{0}$ is the amalgamation of minimal Heegaard splittings of $M^{2}$ and $M^{0}$, and $g\left(M^{2} \cup_{F^{2}} M^{0}\right)=g\left(M_{2}\right)+g\left(F_{1}\right)$.

Proof. The proof is analogous to the proof of Lemma 3.1. The only difference is that $A$ is separating in $F_{1}$ but non-separating in $F_{2}$.

Now suppose that $V \cup_{S} W$ is a minimal Heegaard splitting of $M^{2} \cup_{F^{2}} M^{0}$ with $F_{3} \subset \partial_{-} V$. Since $M^{2} \cup_{F^{2}} M^{0}=\left(F_{1} \times I\right) \cup_{A} M_{2}$, we have $g(S) \leq$ $g\left(M_{2}\right)+g\left(F_{1}\right)$. Following the lines of the proof of Lemma 3.1, $M_{2}$ is not a compression body.

We will show that $V \cup_{S} W$ is weakly reducible. So suppose for contradiction that $V \cup_{S} W$ is strongly irreducible. First note that $S \cap A \neq \emptyset$, since $F^{2}$ is essential in $M$. By Lemma 2.2, we may assume that each component of $S \cap A$ is essential in both $S$ and $A$, and $|S \cap A|$ is minimal.

By proofs similar to those of Claim 1 and 2 we have the following two Claims.

CLAIM 4. $\chi\left(S \cap M_{2}\right) \leq-2 g\left(M_{2}\right)$.

Claim 5. $\chi\left(S \cap\left(F_{1} \times I\right)\right) \leq \chi\left(F_{1}\right)$.

By arguments similar to those in the proof of Lemma 3.1, we know that $S \cap M_{2}$ is bicompressible in $M_{2}$ while $S \cap\left(F_{1} \times I\right)$ is $\partial$-parallel in $F_{1} \times I$. If all components of $S \cap\left(F_{1} \times I\right)$ are parallel to the same component of $F_{1}^{1}$ and $F_{1}^{2}$, say $F_{1}^{1}$, then in $V$, a component of $A \cap V$ cuts off a trivial compression body $F^{1} \times I$ from $V$, but this is impossible since the component of $A \cap V$ is a spanning annulus in $V$. Hence at least one component of $S \cap\left(F_{1} \times I\right)$ is parallel to $F_{1}^{1}$ and at least one component of $S \cap\left(F_{1} \times I\right)$ is parallel to $F_{1}^{2}$. 
Now if $\chi\left(S \cap\left(F_{1} \times I\right)\right)<\chi\left(F_{1}\right)$, then $2 g(S)=2-\chi\left(S \cap M_{2}\right)-\chi(S \cap$ $\left.\left(F_{1} \times I\right)\right)>2 g\left(M_{2}\right)+2 g\left(F_{1}\right)$, a contradiction. Thus $\chi\left(S \cap\left(F_{1} \times I\right)\right)=\chi\left(F_{1}\right)$. Since $A$ is separating in $F_{1}$ and $|\partial A|=2, \chi\left(F_{1}^{1}\right) \leq-1$ and $\chi\left(F_{1}^{2}\right) \leq-1$. Hence if $\chi\left(S \cap\left(F_{1} \times I\right)\right)=\chi\left(F_{1}\right), S \cap\left(F_{1} \times I\right)$ has only two components with one parallel to $F_{1}^{1}$ and the other parallel to $F_{1}^{2}$. This means that $|S \cap A|=2$. Suppose that the boundary components of $A$ are simple closed curves $\alpha_{1}$ and $\alpha_{2}$. Now if $g\left(F_{1}^{1}\right)=r>1$, without loss of generality, we may assume that $\partial F_{1}^{1}=\alpha_{1}$. Take a separating simple closed curve $\gamma$ in $F_{1}^{1}$ such that $F_{1}^{1} \backslash \gamma=T_{1,2} \cup \Sigma_{r-1,1}$, where $T_{1,2}$ is a twice punctured torus with boundary $\alpha_{1}$ and $\gamma$, and $\Sigma_{r-1,1}$ is a once punctured genus $r-1$ surface with boundary $\gamma$. In $M^{2} \cup_{F^{2}} M^{0}$, cut $F_{1}^{1} \times I$ along $\gamma \times I$, we get two 3-manifolds $T_{1,2} \times I$ and $\Sigma_{r-1,1} \times I$. Get rid of $\Sigma_{r-1,1} \times I$ and keep $T_{1,2} \times I$, we add a 2-handle along $\gamma$ to get $\widetilde{M}_{1}=\left(T_{1,2} \times I\right) \cup_{\gamma} 2$-handle. And if $g\left(F_{1}^{1}\right)=1$, we can take $\widetilde{M}_{1}=F_{1}^{1} \times I$. For $F_{1}^{2}$, we do the same operation to get $\widetilde{M}_{2}$. Then we get a 3-manifold $M^{\prime}=\left(\widetilde{M}_{1} \cup_{\alpha_{1} \times I}(A \times I) \cup_{\alpha_{2} \times I} \widetilde{M}_{2}\right) \cup_{A} M_{2}$ (Actually, $\widetilde{M}_{1} \cup_{\alpha_{1} \times I}(A \times I) \cup_{\alpha_{2} \times I} \widetilde{M}_{2}$ is a product of a genus 2 closed surface with I) with a Heegaard surface $S^{\prime}$ induced from $S$. Since $S$ is strongly irreducible, $S^{\prime}$ is strongly irreducible with $g\left(S^{\prime}\right) \leq g\left(M_{2}\right)+2$. Then by Lemma 2.6, $d\left(S_{2}\right) \leq 2 g\left(S^{\prime}\right) \leq 2 g\left(M_{2}\right)+4<2 g\left(M_{2}\right)+5$, a contradiction.

Hence $V \cup_{S} W$ is weakly reducible. Then $V \cup_{S} W$ has an untelescoping as $V \cup_{S} W=\left(V_{1}^{\prime} \cup_{S_{1}^{\prime}} W_{1}^{\prime}\right) \cup_{H_{1}} \ldots \cup_{H_{n-1}}\left(V_{n}^{\prime} \cup_{S_{n}^{\prime}} W_{n}^{\prime}\right)$, where $n \geq 2$, and each component of $H_{i}, 1 \leq i \leq n-1$, is a closed essential surface in $M^{2} \cup_{F^{2}} M^{0}$. Let $\mathscr{H}=\bigcup_{i=1}^{n-1} H_{i}$. We may assume that any component of $\mathscr{H} \cap A$ is essential in both $A$ and $\mathscr{H}$, and $|\mathscr{H} \cap A|$ is minimal. Let $\mathscr{C}^{\prime}=\bigcup H_{i}$ where $H_{i} \in \mathscr{H}$ and $H_{i} \cap M_{2}$ is essential in $M_{2}$. If some component $H^{\prime}$ of $\mathscr{H}^{\prime}$ and $F^{1}$ cobound a Heegaard splitting in the untelescoping, we denote the Heegaard splitting between $H^{\prime}$ and $F^{1}$ by $N_{j}=V_{j}^{\prime} \cup_{S_{j}^{\prime}} W_{j}^{\prime}$. Since $H^{\prime} \cap M_{2}$ is essential in $M_{2}$, by Claim 3 in the proof of Lemma 3.1, we have $\chi\left(S_{j}^{\prime} \cap M_{2}\right) \leq-3-2 g\left(M_{2}\right)$, and by Claim $5, \chi\left(S_{j}^{\prime} \cap\left(F_{1} \times I\right)\right) \leq \chi\left(F_{1}\right)$. Then we have $2 g(S) \geq 4-\chi\left(S_{j}^{\prime}\right) \geq$ $2 g\left(M_{2}\right)+2 g\left(F_{1}\right)+5$, a contradiction.

Hence the outermost component with $H \cap A \neq \emptyset$ must be $\partial$-parallel in $M_{2}$. We may assume that $H$ is isotopic to $F_{1}^{*}$. Let $N_{1}=V_{1}^{\prime} \cup_{S_{1}^{\prime}} W_{1}^{\prime}$ be the Heegaard splitting bounded by $F^{1}, F_{1}^{*}$ and $F_{3}$ in the untelescoping. Then $g\left(N_{1}\right) \geq \min \left\{g\left(F_{1}\right)+g\left(F_{1}^{*}\right), g\left(F_{1}\right)+g\left(F_{3}\right), g\left(F_{1}^{*}\right)+g\left(F_{3}\right)\right\}$. Note that $g\left(F_{3}\right)=g\left(F_{1}\right)+g\left(F_{2}\right)-1$ and $g\left(F_{1}^{*}\right)=g\left(F_{2}\right)+2 g\left(F_{1}^{1}\right)-1$, hence $g\left(S_{1}^{\prime}\right) \geq g\left(N_{1}\right) \geq g\left(F_{1}\right)+g\left(F_{2}\right)$.

If there is no other component of $\mathscr{H}$, we denote the Heegaard splitting in the untelescoping bounded by $F_{1}^{*}$ by $N_{2}=V_{2}^{\prime} \cup_{S_{2}^{\prime}} W_{2}^{\prime}$. $A$ is essential in $M$, and hence in $N_{2}$. Note that $N_{2} \cap M_{1} \cong F_{1}^{1} \times I$ and $N_{2} \cap M_{2} \cong M_{2}$. By Claim 4, we have $\chi\left(S_{2}^{\prime} \cap M_{2}\right) \leq-2 g\left(M_{2}\right)$. Since $S_{2}^{\prime}$ is separating in $N_{2}$, $\left|S_{2}^{\prime} \cap A\right|$ is even while $\left|\partial F_{1}^{1}\right|=1$. This means that $S_{2}^{\prime} \cap\left(F_{1} \times I\right)$ has at least 
two components. Then by Claim $5, \chi\left(S_{2}^{\prime} \cap\left(F_{1} \times I\right)\right) \leq 2 \chi\left(F_{1}^{1}\right)$. Then we have $g(S)=g\left(S_{1}^{\prime}\right)+g\left(S_{2}^{\prime}\right)-g\left(F_{1}^{*}\right) \geq g\left(M_{2}\right)+g\left(F_{1}\right)+1$, a contradiction.

Hence there is some other component $F^{*}$ of $\mathscr{H}$. If $F^{*} \cap M_{2}$ is essential in $M_{2}$, we denote the Heegaard splitting in the untelescoping between $F_{1}^{*}$ and $F^{*}$ by $N_{2}=V_{2}^{\prime} \cup_{S_{2}^{\prime}} W_{2}^{\prime}$. Then by Claim 3 in the proof of Lemma 3.1, we have $\chi\left(S_{2}^{\prime} \cap M_{2}\right) \leq-3-2 g\left(M_{2}\right)$. By Claim 5, we have $\chi\left(S_{2}^{\prime} \cap\left(F_{1} \times I\right)\right) \leq 2 \chi\left(F_{1}^{1}\right)$. Then $g(S)=g\left(S_{1}^{\prime}\right)+g\left(S_{2}^{\prime}\right)-g\left(F_{1}^{*}\right) \geq g\left(M_{2}\right)+g\left(F_{1}\right)+2$, a contradiction.

Hence one component of $\mathscr{H}$ must be parallel to $F^{2}$ in $M_{2}$. Then by the same arguments as in the last paragraph of the proof of Lemma 3.1, only replacing $M_{1}$ by $M_{2}$, we have that $V \cup_{S} W$ is the amalgamation of minimal Heegaard splittings of $M^{2}$ and $M^{0}$, and $g(S)=g\left(M_{2}\right)+g\left(F_{1}\right)$.

Now we come to the proof of Theorem 1.1.

Proof of Theorem 1.1. Let $V \cup_{S} W$ be a minimal Heegaard splitting of $M$. Then $g(S) \leq g\left(M_{1}\right)+g\left(M_{2}\right)$.

If $V \cup_{S} W$ is strongly irreducible, then by Lemma 2.2 we may assume $S \cap A$ is a collection of essential simple closed curves on both $S$ and $A$, and $|S \cap A|$ is minimal. By Claim 1 in the proof of Lemma 3.1, $\chi\left(S \cap M_{1}\right) \leq-2 g\left(M_{1}\right)$, and by Claim 4 in the proof of Lemma 3.2, we have that $\chi\left(S \cap M_{2}\right) \leq-2 g\left(M_{2}\right)$. Then $2 g(S)=2-\chi\left(S \cap M_{1}\right)-\chi\left(S \cap M_{2}\right) \geq 2 g\left(M_{1}\right)+2 g\left(M_{2}\right)+2$, a contradiction.

Hence $V \cup_{S} W$ is weakly reducible. Thus $V \cup_{S} W$ has an untelescoping as

$$
V \cup_{S} W=\left(V_{1}^{\prime} \cup_{S_{1}^{\prime}} W_{1}^{\prime}\right) \cup_{H_{1}} \ldots \cup_{H_{m-1}}\left(V_{m}^{\prime} \cup_{S_{m}^{\prime}} W_{m}^{\prime}\right),
$$

where $m \geq 2$, and for each $i$, each component of $H_{i}$ is a closed essential surface in $M$. Let $\mathscr{F}=\bigcup_{i=1}^{m-1} H_{i}$.

Claim 6. For any $i \in\{2, \ldots, m-1\}$, there are no two components $H_{i-1}$, $H_{i}$ of $\mathscr{F}$ so that $H_{i-1} \cap M_{1}$ is essential in $M_{1}$ and $H_{i} \cap M_{2}$ is essential in $M_{2}$ whether $H_{i-1} \cap M_{1}$ and $H_{i} \cap M_{2}$ have boundary or not.

Proof. Suppose there exist two components of $\mathscr{F}$ so that $H_{i-1} \cap M_{1}$ is essential in $M_{1}$ and $H_{i} \cap M_{2}$ is essential in $M_{2}$. Suppose $V_{i}^{\prime} \cup_{S_{i}^{\prime}} W_{i}^{\prime}$ is the Heegaard splitting in the untelescoping between them. Then by Claim 3 in the proof of Lemma 3.1, we have $\chi\left(S_{i}^{\prime} \cap M_{1}\right) \leq-3-2 g\left(M_{1}\right)$, and $\chi\left(S_{i}^{\prime} \cap\right.$ $\left.M_{2}\right) \leq-3-2 g\left(M_{2}\right)$. Hence $2 g(S) \geq 4-\chi\left(S_{i}^{\prime}\right)>2 g\left(M_{1}\right)+2 g\left(M_{2}\right)+4$, a contradiction.

This completes the proof of Claim 6.

We now divide the proof into the following three cases.

Case 1. No component of $\mathscr{F}$ is $\partial$-parallel in $M_{1}$ or $M_{2}$, and $A \cap \mathscr{F}=\emptyset$. 
In this case, by Claim 6 and the assumption, we may assume that any component of $\mathscr{F}$ is contained in $M_{1}$. Let $H$ be an outermost component of $\mathscr{F}$ in $M_{1}$. By the hypothesis of Case $1, H$ is essential in $M_{1}$.

Suppose $A \subset N_{j}=V_{j}^{\prime} \cup_{S_{j}^{\prime}} W_{j}^{\prime} . A$ is essential in $M$, and hence in $N_{j}$. Since $H$ is essential in $M_{1}$, by Claim 3 in the proof of Lemma 3.1, we have $\chi\left(S_{j}^{\prime} \cap M_{1}\right) \leq-3-2 g\left(M_{1}\right)$. Now $N_{j} \cap M_{2}=M_{2}$, and by Claim 4 in the proof of Lemma 3.2, we have that $\chi\left(S_{j}^{\prime} \cap M_{2}\right) \leq-2 g\left(M_{2}\right)$. Then $2 g(S) \geq$ $4-\chi\left(S_{j}^{\prime}\right) \geq 2 g\left(M_{1}\right)+2 g\left(M_{2}\right)+7$, a contradiction.

Thus, Case 1 cannot happen.

Case 2. No component of $\mathscr{F}$ is $\partial$-parallel in $M_{1}$ or $M_{2}$, and $A \cap \mathscr{F} \neq \emptyset$.

In this case, we may assume that any component of $\mathscr{F} \cap A$ is essential in both $A$ and $\mathscr{F}$, and $|\mathscr{F} \cap A|$ is minimal. There are three subcases.

Subcase 2.1. The outermost component $H$ of $\mathscr{F}$ with $H \cap A \neq \emptyset$ is essential in $M_{1}$ but $\partial$-parallel in $M_{2}$.

By Claim 6, we may assume that each component of $\mathscr{F} \cap M_{1}$ with boundary is essential in $M_{1}$ and each component of $\mathscr{F} \cap M_{2}$ with boundary is $\partial$-parallel in $M_{2}$. Among the components of $\mathscr{F} \cap M_{2}$, let $B$ be the innermost one, that is, $B$ cuts $M_{2}$ into two pieces $M_{2}^{\prime}$ and $M_{2}^{\prime \prime}$, where $M_{2}^{\prime} \cong M_{2}$ and $M_{2}^{\prime \prime} \cong B \times I$, and the interior of $M_{2}^{\prime}$ contains no component of $\mathscr{F} \cap M_{2}$. Let $H_{r}$ be the component of $\mathscr{F}$ containing $B$. Then $H_{r} \cap M_{1}$ is essential in $M_{1}$ and $H_{r} \cap M_{2}$ is $\partial$-parallel in $M_{2}$, see Figure 1 .

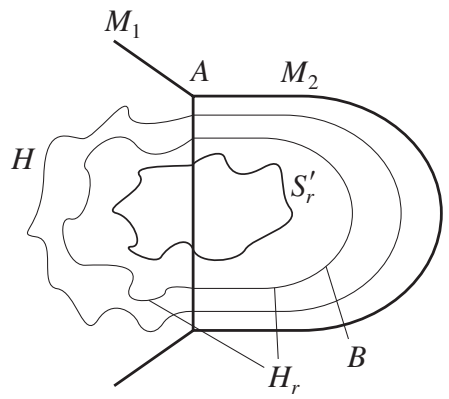

Figure 1.

We may assume that $M_{2}^{\prime}$ is contained in the submanifold $N_{r}=V_{r}^{\prime} \cup_{S_{r}^{\prime}} W_{r}^{\prime}$ of the untelescoping. Since $B$ is innermost, $N_{r}$ is not a product. $V_{r}^{\prime} \cup_{S_{r}^{\prime}} W_{r}^{\prime}$ is a strongly irreducible Heegaard splitting of $N_{r}$. Since any component of $H_{r} \cap M_{1}$ is essential in $M_{1}$, by Claim 3 in the proof of Lemma 3.1, $\chi\left(S_{r}^{\prime} \cap M_{1}\right) \leq$ $-3-2 g\left(M_{1}\right)$. Note that $N_{r} \cap M_{2} \cong M_{2}$, and by Claim 4 in the proof of Lemma 3.2, we have $\chi\left(S_{r}^{\prime} \cap M_{2}\right) \leq-2 g\left(M_{2}\right)$. Then $2 g(S) \geq 4-\chi\left(S_{r}^{\prime}\right) \geq$ $2 g\left(M_{1}\right)+2 g\left(M_{2}\right)+7$, a contradiction. 
Subcase 2.2. The outermost component $H$ of $\mathscr{F}$ with $H \cap A \neq \emptyset$ is essential in $M_{2}$ but $\partial$-parallel in $M_{1}$.

There are two sub-subcases.

Sub-subcase 2.2.1. Each component of $H \cap M_{1}$ is parallel to the same one of $F_{1}^{1}$ or $F_{1}^{2}$, say $F_{1}^{1}$, in $M_{1}$.

We denote the Heegaard splitting in the untelescoping between $F_{3}$ and $H$ by $N_{j}=V_{j}^{\prime} \cup_{S_{j}^{\prime}} W_{j}^{\prime}$. See Figure 2 . Note that by Clai $6, N_{j} \cap M_{1} \cong M_{1}$, and by Claim 1 in the proof of Lemma 3.1, we have that $\chi\left(S_{j}^{\prime} \cap M_{1}\right) \leq-2 g\left(M_{1}\right)$. Since $H \cap M_{2}$ is essential in $M_{2}$, by Claim 3 in the proof of Lemma 3.1, $\chi\left(S_{j}^{\prime} \cap M_{2}\right) \leq-3-2 g\left(M_{2}\right)$. Then $2 g(S) \geq 4-\chi\left(S_{j}^{\prime}\right) \geq 2 g\left(M_{1}\right)+2 g\left(M_{2}\right)+7$, a contradiction.

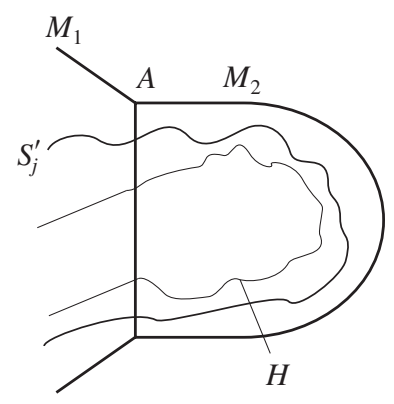

FIGURE 2.

Sub-subcase 2.2.2. At least one component of $H \cap M_{1}$ is parallel to $F_{1}^{1}$ and at least one component of $H \cap M_{1}$ is parallel to $F_{1}^{2}$ in $M_{1}$.

By Claim 6, we may assume that each component of $\mathscr{F} \cap M_{1}$ with boundary is $\partial$-parallel in $M_{1}$. Among the components of $\mathscr{F} \cap M_{1}$ which are parallel to $F_{1}^{1}$, let $B_{1}$ be the innermost one, and among the components of $\mathscr{F} \cap M_{1}$ which are parallel to $F_{1}^{2}$, let $B_{2}$ be the innermost one, that is, $B_{1}$ and $B_{2}$ cut $M_{1}$ into three pieces $M_{1}^{\prime}, M_{1}^{\prime \prime}$ and $M_{1}^{\prime \prime \prime}$ with $M_{1}^{\prime} \cong B_{1} \times I, M_{1}^{\prime \prime} \cong M_{1}$ and $M_{1}^{\prime \prime \prime} \cong B_{2} \times I$, and the interior of $M_{1}^{\prime \prime}$ contains no component of $\mathscr{F} \cap M_{1}$. By swapping the labels of $B_{1}$ and $B_{2}$ if necessary, we may suppose that the number of components of $\mathscr{F} \cap M_{1}$ in $M_{1}^{\prime}$ is greater than the number in $M_{1}^{\prime \prime \prime}$. Let $H_{j}$ be the component of $\mathscr{F}$ containing $B_{2}$. Then by Claim 6, we have that $H_{j} \cap M_{1}$ is $\partial$-parallel in $M_{1}$ and $H_{j} \cap M_{2}$ is essential in $M_{2}$. See Figure 3.

We may assume that $M_{1}^{\prime \prime}$ is contained in the submanifold $N_{j}=V_{j}^{\prime} \cup_{S_{j}^{\prime}} W_{j}^{\prime}$ of the untelescoping. Since $H_{j} \cap M_{2}$ is essential in $M_{2}$, by Claim 3 in the proof of Lemma 3.1, $\chi\left(S_{j}^{\prime} \cap M_{2}\right) \leq-3-2 g\left(M_{2}\right)$. Note that $N_{j} \cap M_{1} \cong M_{1}$, and by Claim 1 in the proof of Lemma 3.1, we have that $\chi\left(S_{j}^{\prime} \cap M_{1}\right) \leq-2 g\left(M_{1}\right)$. Then $2 g(S) \geq 4-\chi\left(S_{j}^{\prime}\right) \geq 2 g\left(M_{1}\right)+2 g\left(M_{2}\right)+7$, a contradiction. 


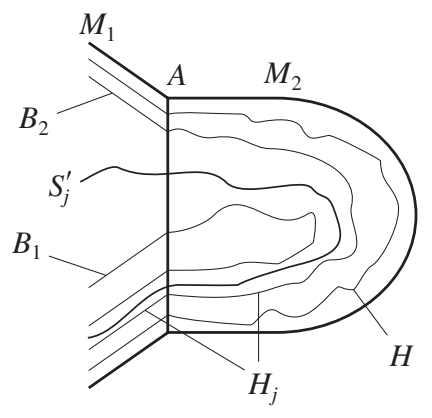

Figure 3.

Subcase 2.3. The outermost component $H$ of $\mathscr{F}$ with $H \cap A \neq \emptyset$ is isotopic to $F_{1}^{*}$ or $F_{2}^{*}$, say $F_{1}^{*}$.

We denote the Heegaard splitting in the untelescoping between $F_{3}$ and $F_{1}^{*}$ by $N_{j}=V_{j}^{\prime} \cup_{S_{j}^{\prime}} W_{j}^{\prime}$. Let $S_{j}^{1}=S_{j}^{\prime} \cap M_{1}$ and $S_{j}^{2}=S_{j}^{\prime} \cap M_{2}$. Now if $N_{j}$ has some other boundary component $H^{*}$, then by assumption $H^{*} \cap A=\emptyset$, i.e., $H^{*}$ is a closed essential surface in $M_{1}$ or $M_{2}$. Now $N_{j} \cap M_{2} \cong\left(F_{2}-i n t A\right) \times I$, hence $H^{*} \subset M_{1}$. Since $H^{*}$ is an essential surface in $M_{1}$, by Claim 3 and Claim 2 in the proof of Lemma 3.1, we have that $\chi\left(S_{j}^{1}\right) \leq-3-2 g\left(M_{1}\right), \chi\left(S_{j}^{2}\right) \leq$ $\chi\left(F_{2}\right)$. If $N_{j}$ has no other boundary component, then $N_{j} \cap M_{1} \cong M_{1}$. By Claim 1 and Claim 2 in the proof of Lemma 3.1, we have $\chi\left(S_{j}^{1}\right) \leq-2 g\left(M_{1}\right)$, $\chi\left(S_{j}^{2}\right) \leq \chi\left(F_{2}\right)$. Hence whether $N_{j}$ has some other boundary component or not, we have $\chi\left(S_{j}^{\prime}\right)=\chi\left(S_{j}^{1}\right)+\chi\left(S_{j}^{2}\right) \leq 2-2 g\left(M_{1}\right)-2 g\left(F_{2}\right)$.

We denote the Heegaard splitting in the untelescoping on the other side of $F_{1}^{*}$ which has $F_{1}^{*}$ as a boundary component by $N_{r}=V_{r}^{\prime} \cup_{S_{r}^{\prime}} W_{r}^{\prime}$. Let $S_{r}^{i}=S_{r}^{\prime} \cap M_{i}$, $i=1,2$.

There are three sub-subcases.

Sub-subcase 2.3.1. $N_{r}$ has another boundary component $H^{\prime}$ of $\mathscr{F}$ with $H^{\prime} \cap M_{1}$ essential in $M_{1}$.

In this case, if $H^{\prime} \cap M_{2}=\emptyset$, then $H^{\prime} \subset\left(F_{1}^{1} \times I\right)$, which means that a compression body contains a closed essential surface, a contradiction. Hence $H^{\prime} \cap M_{2} \neq \varnothing$, and all components of $H^{\prime} \cap M_{2}$ are $\partial$-parallel in $M_{2}$, and furthermore, by Claim 6, we may assume that each component of $\left(\mathscr{F}-F_{1}^{*}\right) \cap$ $M_{1}$ with boundary is essential in $M_{1}$ and each component of $\mathscr{F} \cap M_{2}$ with boundary is $\partial$-parallel in $M_{2}$.

The following arguments are in some sense similar to those in Subcase 2.1. Take the innermost component $B$ of $\mathscr{F} \cap M_{2}$, that is, $B$ cuts $M_{2}$ into two pieces $M_{2}^{\prime}$ and $M_{2}^{\prime \prime}$, where $M_{2}^{\prime} \cong M_{2}$ and $M_{2}^{\prime \prime} \cong B \times I$, and the interior of $M_{2}^{\prime}$ contains no component of $\mathscr{F} \cap M_{2}$. Let $H_{i}$ be the component of $\mathscr{F}$ containing 
$B$. Then $H_{i} \cap M_{1}$ is essential in $M_{1}$ and $H_{i} \cap M_{2}$ is $\partial$-parallel in $M_{2}$. We may assume that $M_{2}^{\prime}$ is contained in the submanifold $N_{i}=V_{i}^{\prime} \cup_{S_{i}^{\prime}} W_{i}^{\prime}$ of the untelescoping. $V_{i}^{\prime} \cup_{S_{i}^{\prime}} W_{i}^{\prime}$ is a strongly irreducible Heegaard splitting of $N_{i}$. Since $H_{i} \cap M_{1}$ is essential in $M_{1}$, by Claim 3 in the proof of Lemma 3.1, $\chi\left(S_{i}^{\prime} \cap M_{1}\right) \leq-3-2 g\left(M_{1}\right)$. Note that $N_{i} \cap M_{2} \cong M_{2}$, and by Claim 4 in the proof of Lemma 3.2, $\chi\left(S_{i}^{\prime} \cap M_{2}\right) \leq-2 g\left(M_{2}\right)$. Then $2 g(S) \geq 4-\chi\left(S_{i}^{\prime}\right) \geq$ $2 g\left(M_{1}\right)+2 g\left(M_{2}\right)+7$, a contradiction.

Sub-subcase 2.3.2. $N_{r}$ has another boundary component $H^{\prime}$ of $\mathscr{F}$ with $H^{\prime} \cap M_{2}$ essential in $M_{2}$.

In this case, $H^{\prime} \cap M_{2}$ essential in $M_{2}$, and by Claim 3 in the proof of Lemma 3.1, we have that $\chi\left(S_{r}^{2}\right) \leq-3-2 g\left(M_{2}\right)$. Whether $H^{\prime} \cap M_{1}=\varnothing$ or not, see Figure $4(a),(b)$, by Claim 5 in the proof of Lemma 3.2, we have that $\chi\left(S_{r}^{1}\right) \leq 2 \chi\left(F_{1}^{1}\right)$. Hence $2 g(S) \geq 2-\chi\left(S_{r}^{\prime}\right)-\chi\left(S_{j}^{\prime}\right)+\chi\left(F_{1}^{*}\right) \geq$ $2 g\left(M_{1}\right)+2 g\left(M_{2}\right)+5$, a contradiction.

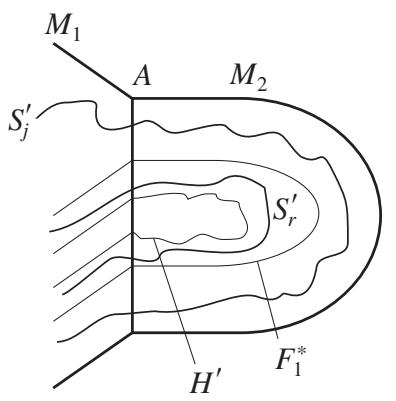

(a)

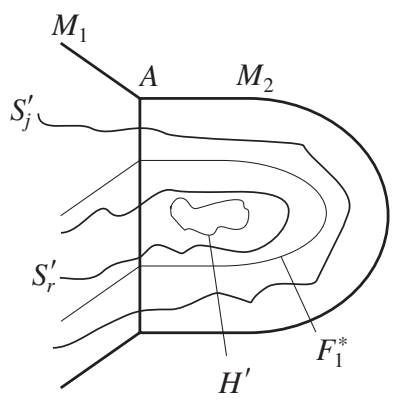

(b)

FIGURE 4.

Sub-subcase 2.3.3. $N_{r}$ has no other boundary component.

In this case, $N_{r} \cap M_{2} \cong M_{2}$, see Figure 5. By Claim 4 and Claim 5 in the proof of Lemma 3.2, we have that $\chi\left(S_{r}^{1}\right) \leq 2 \chi\left(F_{1}^{1}\right), \chi\left(S_{r}^{2}\right) \leq-2 g\left(M_{2}\right)$. Hence $2 g(S)=2-\chi\left(S_{r}^{\prime}\right)-\chi\left(S_{j}^{\prime}\right)+\chi\left(F_{1}^{*}\right) \geq 2 g\left(M_{1}\right)+2 g\left(M_{2}\right)+2$, a contradiction.

Therefore, Case 2 cannot happen. Thus, we have only one possibility.

Case 3. There exists one component of $\mathscr{F}$ which is $\partial$-parallel in $M_{1}$ or $M_{2}$. By applying Lemma 3.1 and Lemma 3.2 to this case, we have the stated conclusion.

This finishes the proof of Theorem 1.1. 


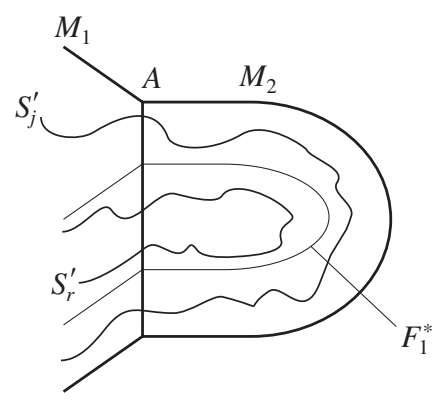

FIGURE 5.

Proposition 3.3. Any minimal Heegaard splitting of $M^{0}$ is strongly irreducible.

Proof. Let $V_{0} \cup_{S_{0}} W_{0}$ be any minimal Heegaard splitting of $M^{0}$. Since $M^{0}=F_{1} \times I \cup_{A} F_{2} \times I$, from the result of Schultens, we have $g\left(S_{0}\right) \leq g\left(F_{1}\right)+$ $g\left(F_{2}\right)$. Since $A$ is separating on $F_{1}$ and non-separating on $F_{2}, M^{0}$ contains only three boundary components $F^{1}, F^{2}$ and $F_{3}=F_{1}^{1} \cup\left(F_{2} \backslash A_{2}\right) \cup F_{1}^{2}$. Note that $g\left(F_{3}\right)=g\left(F_{1}\right)+g\left(F_{2}\right)-1$, and then $g\left(S_{0}\right)=g\left(M^{0}\right) \geq \min \left\{g\left(F^{1}\right)+\right.$ $\left.g\left(F^{2}\right), g\left(F^{1}\right)+g\left(F_{3}\right), g\left(F^{2}\right)+g\left(F_{3}\right)\right\} \geq g\left(F_{1}\right)+g\left(F_{2}\right)$. Hence we have $g\left(S_{0}\right)=g\left(F_{1}\right)+g\left(F_{2}\right)$.

Now if $V_{0} \cup_{S_{0}} W_{0}$ is weakly reducible, then it has an untelescoping as $\left(V_{1}^{\prime} \cup_{S_{1}^{\prime}} W_{1}^{\prime}\right) \cup_{H_{1}}\left(V_{2}^{\prime} \cup_{S_{2}^{\prime}} W_{2}^{\prime}\right)$, where $H_{1}$ is isotopic to either $F_{1}^{*}$ or $F_{2}^{*}$. We may assume that $F_{3} \subset \partial_{-} V_{1}^{\prime}$. Since $F_{3}$ is incompressible in $M^{0}$ and the length of the untelescoping is at least 2 , we have $g\left(S_{0}\right) \geq g\left(S_{1}^{\prime}\right)+1 \geq g\left(F_{3}\right)+2 \geq$ $g\left(F_{1}\right)+g\left(F_{2}\right)+1$, a contradiction. Hence $V_{0} \cup_{S_{0}} W_{0}$ is strongly irreducible.

Now we come to the proof of Theorem 1.2.

Proof of Theorem 1.2. Since $d\left(S_{i}\right) \geq 2 g\left(M_{i}\right)+5$, Lemma $2.7 \mathrm{im}-$ plies that $S_{i}$ is the unique minimal Heegaard splitting of $M_{i}$ for $i=1,2$. By Theorem 1.1, any minimal Heegaard splitting of $M$ is the amalgamation of minimal Heegaard splittings of $M^{1}, M^{0}$, and $M^{2}$ along $F^{1}, F^{2}$, and $g(M)=g\left(M_{1}\right)+g\left(M_{2}\right)$. To prove Theorem 1.2, we only need to consider the minimal Heegaard splitting $V_{0} \cup_{S_{0}} W_{0}$ of $M^{0}$. By Proposition 3.3, $V_{0} \cup_{S_{0}} W_{0}$ is strongly irreducible. $M^{0}$ contains three boundary components $F^{1}, F^{2}$ and $F_{3}$, and the type of $V_{0} \cup_{S_{0}} W_{0}$ is only determined by the partition of its boundary components. We may assume that $F_{3} \subset \partial_{-} V_{0}$. Note that since $A$ is separating on $F_{1}, g\left(F_{1}\right) \geq 2$.

(1) When $g\left(F_{2}\right) \geq 2$, since $g\left(F_{3}\right)=g\left(F_{1}\right)+g\left(F_{2}\right)-1>g\left(F_{1}\right), g\left(F_{2}\right)$, the only possibility is $F_{3}=\partial_{-} V_{0}, F^{1} \cup F^{2}=\partial_{-} W_{0}$. Hence the minimal Heegaard splitting of $M$ is unique. 
(2) When $g\left(F_{2}\right)=1, g\left(F_{3}\right)=g\left(F_{1}\right)+g\left(F_{2}\right)-1=g\left(F_{1}\right)$, then there are two possibilities: $F_{3}=\partial_{-} V_{0}, F^{1} \cup F^{2}=\partial_{-} W_{0}$ or $F^{1}=\partial_{-} V_{0}, F^{2} \cup F_{3}=$ $\partial_{-} W_{0}$. Hence there are at most two minimal Heegaard splittings of $M$.

This completes the proof of Theorem 1.2.

ACKNOWLEDGEMENTS. We wish to thank the referee most warmly for numerous suggestions that have improved the exposition of this paper.

\section{REFERENCES}

1. Casson, A. J., and Gordon, C. M., Reducing Heegaard Splittings, Topology Appl. 27 (1987), 275-283.

2. Du, K., Ma, J., Qiu, R., and Zhang, M., Heegaard genera of annular 3-manifolds, J. Knot Theory Ramifications 20 (2011), 547-566.

3. Hartshorn, K., Heegaard splittings of Haken manifolds have bounded distance, Pacific J. Math. 204 (2002), 61-75.

4. Hempel, J., 3-manifolds as viewed from the curve complex, Topology 40 (2001), 631-657.

5. Jaco, W., Lectures on three manifold topology, CBMS Conference Ser. Math. 43, Amer. Math. Soc., Providence 1980.

6. Kobayashi, T., and Qiu, R., The amalgamation of high distance Heegaard splittings is always efficient, Math. Ann. 341 (2008), 707-715.

7. Lei, F., and Yang, G., A lower bound of genus of amalgamations of Heegaard splittings, Math. Proc. Camb. Phil. Soc. 146 (2009), 615-623.

8. Li, F., Yang, G., and Lei, F., Heegaard genera of high distance are additive under annulus sum, Topology Appl. 157 (2010), 1188-1194.

9. Morimoto, K., Tunnel number, connected sum and meridional essential surface, Topology 39 (2000), 469-485.

10. Scharlemann, M., Local detection of strongly irreducible Heegaard splittings, Topology Appl. 90 (1998), 135-147.

11. Scharlemann, M., Proximity in the curve complex: boundary reduction and bicompressible surfaces, Pacific J. Math. 228 (2006), 325-348.

12. Scharlemann, M., and Schultens, J., The tunnel number of the sum of $n$ knots is at least $n$, Topology 38 (1999), 265-270.

13. Scharlemann, M., and Thompson, A., Thin position for 3-manifolds, pp. 231-238 in: Geometric topology, Contemp. Math. 164, Amer. Math. Soc., Providence 1994.

14. Scharlemann, M., and Tomova, M., Alternate Heegaard genus bounds distance, Geom. Topol. 10 (2006), 593-617.

15. Schultens, J., Additivity of tunnel number for small knots, Comment. Math. Helv. 75 (2000), 353-363.

16. Schultens, J., The classification of Heegaard splittings for (compact orientable surfaces) $\times S^{1}$, Proc. London Math. Soc. 67 (1993), 425-448.

17. Yang, G., and Lei, F., Some sufficient conditions for tunnel numbers of connected sum of two knots not to go down, Acta Math. Sin. (Engl. Ser.) 27 (2011), 2229-2244.

SCHOOL OF MATHEMATICAL SCIENCES

DALIAN UNIVERSITY OF TECHNOLOGY

DALIAN 116024

CHINA

E-mail: dutlf1@163.com,fclei@dlut.edu.cn
DEPARTMENT OF MATHEMATICS HARBIN INSTITUTE OF TECHNOLOGY HARBIN 150001

CHINA

E-mail: gqyang@hit.edu.cn 